\title{
Connaissance, Attitudes et pratiques des élèves sur l'hygiène alimentaire et l'alimentation de la rue
}

\section{Knowledge, Attitudes and practices of students on food hygiene and street food}

\author{
Soumahoro $\mathrm{SI}^{1,2}$; Coulibaly $\mathbf{M}^{1}$, Kouassi $\mathrm{DP}^{1,2}$, Irika $\mathrm{O}^{1}$, Goua $\mathrm{Bi}^{1}$, Ouaga $\mathrm{JM}^{1}$, Angbo-Effi $\mathrm{O}^{2}$ \\ 1 Antenne Régionale de l'Institut National d'Hygiène Publique de Bouaké, \\ 2 Département de Santé publique et maladie infectieuse, Université Alassane Ouattara de Bouaké \\ Correspondant : COULIBALY M'bégnan ; Institut National d'Hygiène Publique (INHP) de Bouaké ; \\ Côte d'Ivoire Email : $\underline{\text { m begnan@yahoo.fr }}$
}

\begin{abstract}
Résumé
Introduction : Dans les pays en développement, les risques de contamination des aliments sont à la fois présents à domicile que chez les vendeurs mais avec un risque de contamination plus élevé des aliments de la rue. Au regard des risques encourus par la transmission de maladies par les aliments, nous avons mené cette étude auprès des élèves de Bouaké afin d'apprécier leurs connaissances, attitudes et pratiques vis-à-vis de l'hygiène des aliments vendus dans les rues dans le but d'améliorer les comportements en hygiène alimentaire des élèves.
\end{abstract}

Méthode: Nous avons mené une étude transversale à visée descriptive dans quatre établissements secondaires, dont deux publics et deux privés, de la ville de Bouaké. Nous avons interviewé 448 élèves grâce à un questionnaire. Le logiciel SPSS 17.0 a été utilisé pour la saisie et l'analyse des données recueillies.

Résultats : Les élèves interrogés avaient un âge médian de $16 \pm 5,2$ ans, étaient majoritairement de sexe masculin $(60,0 \%)$ et percevaient de l'argent de poche dans $92,2 \%$ des cas. Nos enquêtés affirmaient qu'il fallait se laver les mains avec de l'eau et du savon $(99,1 \%)$ et se lavaient systématiquement les mains avant chaque repas $(94,9 \%)$. Ils ne faisaient pas confiance aux aliments vendus à l'école ou dans la rue $(64,1 \%)$ et certains élèves interrogés disaient être tombés malades après consommation d'aliments vendus dans la rue $(53,6 \%)$.

Conclusion : Le respect des pratiques d'hygiène alimentaire est le garant de la prévention de nombreuses maladies infectieuses. Des dispositions en rapport avec un meilleur contrôle de l'alimentation de rue devraient être prises pour minimiser le risque de survenue des maladies infectieuses liées à l'Alimentation de la rue.

Mots-clés : Alimentation de rue - Elève Hygiène alimentaire

\footnotetext{
Abstract

Introduction: In developing countries, the risks of food contamination are both present at home and at the vendors' premises but with a higher risk
}

of contamination of street food. In view of the risks involved in the transmission of diseases through food, we are conducting this study among the students of Bouaké in order to assess their knowledge, attitudes and practices regarding the hygiene of food sold on the streets.

Method: We conducted a descriptive crosssectional study in four secondary schools, two public and two private, in the city of Bouaké. We interviewed 448 students through a questionnaire. SPSS 17.0 software was used for data collection and analysis.

Results: The students surveyed had a median age of $16 \pm 5.2$ years, were predominantly male $(60.0 \%)$ and received pocket money in $92.2 \%$ of cases. Our respondents said that they should wash their hands with soap and water (99.1\%) and systematically washed their hands before every single meal $(94.9 \%)$. They did not trust food sold at school or on the street $(64.1 \%)$, and some of them said they became sick after eating street foods $(53.6 \%)$.

Conclusion: Good food hygiene practices are guarantees for the prevention of many infectious diseases. Provisions for better street food control should be taken to minimize infectious diseases due to street food.

Key-words: Street food - Student - Food hygiene

\section{Introduction}

Les aliments sont les principales voies de transmission des maladies à causes des risques de contamination microbienne [1]. Les pays en développement sont les plus touchés par les maladies liées à la nourriture [2,3]. Ces maladies qui causent environ 700.000 décès en Afrique chaque année, sont sous-notifiées [4]. Les germes tels que le Campylobacter [5,6], la Shighelle ou Escherichia coli [7] sont responsables de la contamination des aliments et provoquent des épidémies. Dans ces pays pauvres, au niveau d'hygiène précaire et un accès limité à l'eau potable, les maladies liées à la mauvaise hygiène constituent un véritable problème de santé publique [8], notamment celles liées à la 
mauvaise hygiène des aliments $[\mathbf{9 , 1 0}]$. Dans les pays en développement, les risques de contamination des aliments sont à la fois présents à domicile [11] que chez les vendeurs [12] mais avec une contamination plus élevée des aliments de la rue $[13,14]$. Par ailleurs la majorité des vendeurs des aliments de rue connaissent les mesures d'hygiène alimentaire mais négligent leur mise en pratique [14]. Nombreuses études menées, notamment auprès des étudiants du premier cycle d'âge moyen de 18 ans à travers le monde ont montré un manque de connaissances appropriées sur la sécurité des aliments [15-20]. En Côte d'Ivoire, peu d'études ont été menées en hygiène alimentaire, notamment dans les établissements scolaires. A l'instar des autres villes de Côte d'Ivoire, Bouaké, deuxième ville de Côte d'Ivoire, connait des problèmes de salubrité. $\mathrm{Au}$ regard des risques encourus par la transmission des maladies par les aliments, nous avons mené cette étude auprès des élèves de Bouaké afin d'apprécier leurs connaissances, attitudes et pratiques vis-à-vis de l'hygiène des aliments vendus dans les rues. Cette étude a pour but d'améliorer les comportements en hygiène alimentaire des élèves de la ville de Bouaké.

\section{Méthodologie}

Nous avons mené une étude transversale à visée descriptive dans quatre établissements secondaires, dont deux publics et deux privés, de la ville de Bouaké.

La taille d'échantillon des élèves à enquêter, N, a été calculée selon la formule de SCHWARTZ, $\mathbf{N}$ $=\mathbf{t}^{2} \mathbf{p}(\mathbf{1}-\mathbf{p}) / \mathbf{i}^{2}$, avec :

$\mathbf{N}$ : la taille minimale de l'échantillon nécessaire. t: valeur seuil lue dans le test de l'écart réduit pour $\mathbf{a}=5 \% \quad \mathbf{p}$ : la prévalence estimée, $\mathbf{p}=50 \%$

i : la précision de l'estimation (5\%) Alors, $\mathrm{N}=(1,96){ }^{2}(0,5)(0,5) / 0.05^{2} \approx 384$ élèves. Avec un taux de non réponse à $10 \%$, la taille d'échantillon retenue pour l'étude était donc de 424 élèves. Les élèves à enquêter ont été sélectionnés après un échantillonnage randomisé à trois degrés, dont le premier était les établissements, le second, une seule classe parmi toutes les classes de chaque niveau scolaire de l'établissement sélectionné, et enfin le troisième était les élèves des classes sélectionnées. Le nombre d'élèves par niveau d'étude a été déterminé à proportions égales du nombre de niveaux d'étude des établissements secondaires, c'est-à-dire 7 niveaux; soit environ 16 élèves à recruter par niveau d'étude.

Les données ont été collectées à partir d'un questionnaire lors d'interview individuelles en face à face dans les établissements scolaires, aux heures de pause afin d'éviter toute gène au bon déroulement des cours. Les données ont été analysées à l'aide du logiciel SPSS 17.0.

\section{Considération éthique}

En vertu de la loi sur les pratiques des enquêtes, nous avons protégé la confidentialité par l'attribution d'un numéro d'anonymat sur le questionnaire de chaque élève enquêté. Par ailleurs la participation des enquêtés était libre et obtenue après consentement éclairé et accord verbal. Par ailleurs, une autorisation préalable a été obtenue auprès des autorités des deux Directions Régionales de l'Education Nationale de Bouaké.

Résultats

\section{Caractéristiques sociodémographiques des} enquêtés

Au terme de notre enquête, nous avons interrogé 448 élèves, d'âge médian de $16 \pm 5,2$ ans et de tranche d'âge compris entre 15 et 19 ans dans $51,8 \%$ des cas. Ils étaient de sexe masculin $(60,0 \%)$. Parmi eux, 84,4\% vivaient avec leurs parents et $92,2 \%$ percevaient de l'argent de poche (Tableau I).

Connaissances des élèves sur le lavage des mains, la gestion des déchets alimentaires, les maladies liées à la mauvaise hygiène alimentaire

Nos enquêtés affirmaient qu'il fallait se laver les mains avec de l'eau et du savon $(99,1 \%)$, mais certains pensaient que le lavage des mains se faisait en plongeant les mains dans un récipient $(47,1 \%)$. Ils affirmaient que les déchets d'origine alimentaire devraient être stockés dans des poubelles à couvercle $(87,3 \%)$. Ils connaissaient des maladies liées à la mauvaise hygiène alimentaire comme le choléra $(54,9 \%)$ et la fièvre typhoïde $(58,7 \%)$ (Tableau II).

Pratiques des élèves en hygiène des mains

Les enquêtés affirmaient se laver systématiquement les mains avant chaque repas $(94,9 \%)$. Ils pratiquaient le lavage des mains avec de l'eau et du savon $(85,3 \%)$, pendant 30 secondes à 1 minutes dans $61,2 \%$ des cas, en plongeant les mains dans un récipient $(45,8 \%)$ (Tableau III).

Attitudes des élèves des aliments vendus dans la rue

Les élèves enquêtés trouvaient malsains les aliments vendus à proximité d'ordures $(87,7 \%)$, d'eaux usées $(93,5 \%)$, de caniveaux ouverts $(94,6 \%)$ ou la présence de nuisibles à proximité des points de vente d'aliments $(94,0 \%)$. Ils ne faisaient pas confiance aux aliments vendus à l'école ou dans la rue $(64,1 \%)$. Ils affirmaient consommer les aliments vendus non couverts $(54,0 \%)$, vendus à proximité des caniveaux à ciel ouvert $(21,7 \%)$, des ordures ménagères $(17,0 \%)$ et des eaux usées $(10,9 \%)$. Certains enquêtés 
disaient être tombés malades après consommation d'aliments vendus dans la rue $(53,6 \%)$ (Tableau IV).

\section{Discussion}

Cette étude menée auprès de 448 élèves de quatre établissements secondaires de Bouaké a permis d'enquêter des élèves de sexe masculin pour la plupart (60\%). Cette prédominance masculine des élèves reflète la disparité de genre dans la scolarisation en Côte d'Ivoire [21]. L'âge médian des enquêtés était de 16 ans, qui s'expliquerait par le nombre plus élevé de niveaux d'étude au $1^{\text {er }}$ cycle d'étude (4 niveaux) qu'au second cycle (3 niveaux). Leur connaissance sur le lavage des mains montrait que, bien que la quasi-totalité d'entre eux sache qu'il fallait de l'eau et du savon pour accomplir un bon lavage des mains $(99,1 \%)$, ils ignoraient les bonnes pratiques. Ils pensaient que l'on pouvait utiliser que de l'eau (14,74\%), plonger directement les mains dans un récipient $(47,08 \%)$ ou se laver correctement les mains en moins d'une minute $(66,8 \%)$. Le lavage correct des mains était aussi méconnu des élèves, dans une étude à Seoul et Ulsan [22]. Pourtant la charge bactérienne des mains est réduite à $23 \%$ après le lavage des mains à l'eau seule et à $8 \%$ après le lavage des mains à l'eau et au savon [23]. Le lavage des mains dans un même récipient ne permettrait pas l'élimination des germes lors du rinçage ou même conduirait à une récontamination des mains. La méthode de lavage des mains devrait être enseignée pour réduire la transmission des maladies manu-portées. Le lavage des mains permet de réduire le risque de diarrhée lié aux maladies infectieuses de $50 \%$ et sauve des millions de vies [24]. Le lavage des mains avec du savon avant la confection des mets permet également de réduire le risque de maladies d'origine alimentaire [25]. La disponibilité de l'eau et du savon est fortement associée à une prévalence plus élevée du comportement de lavage des mains [26,27]. Pour la protection des aliments, les enquêtés savaient qu'il fallait stocker les déchets alimentaires dans des poubelles à couvercle $(87,3 \%)$. Ils trouvaient inapproprié la vente d'aliments à proximité de source de pollution diverse (débris alimentaires, décharges d'ordure, eaux usées, caniveaux non couverts, etc.). Pourtant, ils ne trouvaient pas d'inconvénients à consommer des aliments non protégés $(75,9 \%)$. Ces résultats démontraient que nos enquêtés ne seraient préoccupés que par la gêne visuelle ou l'odeur que procureraient les sources de pollution, mais ne percevraient pas le risque de contamination entre ces sources de pollution et les aliments ou même le risque de la prolifération de vecteurs susceptibles de contaminer les aliments. Chauliac M et col ont, dans une étude menée en 1998 à Cotonou, trouvé que les vendeuses dont l'hygiène était jugée non satisfaisante étaient rejetées par $17,5 \%$ des écoliers interrogés. Toujours dans la même étude, les raisons spontanées qui motivaient l'achat fréquent des aliments étaient surtout le bon goût (28,3\%) et la satiété $(27,1 \%)$ [28]. Dans une autre étude de Chauliac M et col menée en 1994 à Bamako au Mali, les écoliers des cours préparatoires et cours moyens achetaient surtout les plats cuits, dans respectivement 60,3 et $65,0 \%$ [29]. Le renforcement de la sensibilisation des élèves devrait leur faire prendre conscience du danger auquel ils s'exposent lors de la consommation d'aliments non couverts, d'autant plus que certains consommaient des aliments vendus aux abords de source de pollution comme les dépôts d'ordure (17\%), les caniveaux à ciel ouvert $(21,7 \%)$ ou même les aliments non protégés $(46,0 \%)$. Les messages de promotion de l'hygiène alimentaire permettraient de parvenir au contrôle définitif des maladies des mains sales.

\section{Conclusion}

De bonnes pratiques d'hygiène alimentaire sont des garanties pour la prévention de nombreuses maladies infectieuses. L'alimentation de rue reste incontrôlée dans de nombreux pays comme la Côte d'Ivoire, qui continue de demeurer sources de maladies pour les populations, notamment des élèves qui en sont des victimes. De meilleures dispositions en rapport avec le contrôle de l'alimentation de rue devraient être prises pour la prévention de ces maladies.

Conflit d'intérêt : Les auteurs déclarent ne pas avoir de conflit d'intérêt en relation avec cet article

\section{Références}

1. Odeyemi OA, Bamidele FA. Harnessing the potentials of predictive microbiology in microbial food safety and quality research in Nigeria. Future Science OA 2016, 2(1): FSO91-93.

2. World Health Organization. Food safety and food borne illness. Geneva: WHO; 2007.

3. World Health Organization. Consultation to Develop a Strategy to Estimate the Global Burden of Foodborne Diseases. Geneva: WHO Consultation; 2006.

4. National Food Safety Systems in Africa. A situation analysis. Harare, Zimbabwe: FAO/WHO Regional Conference on Food Safety for Africa; 2005.

5. Platts-Mills JA and Kosek M. Update on the burden of Campylobacter in developing countries. Curr. Opin. Infect. Dis., 2014, 27(5): 444-450.

6. Wei, B, Cha SY, Yoon RH, Kang M, Roh JH, Seo HS, Lee JA. and Jang HK. (2016) Prevalence and antimicrobial resistance of Campylobacter spp. 
Isolated from retail chicken and duck meat in South Korea. Food Control, 62: 63-68.

7. Murray CJ, Vos T, Lozano R, et al. (2012) Disabilityadjusted life years (DALYs) for 291 diseases and injuries in 21 regions, 1990-2010: A systematic analysis for the Global Burden of Disease Study 2010. Lancet, 380: 2197-2223.

8. Abera G. Shigellosis in Ethiopia: review of studies conducted since 1974. Ethiop J Biol Sci. 2004;3:191-235.

9. Nesbitt A, Majowicz S, Finley R, Marshall B, Pollari F, Sargeant J, et al. High-risk food consumption and food safety practices in a Canadian community. J Food Prot. 2009;72(12): 2575-86.

10. Fein SB, Lando AM, Levy AS, Teisi MF, Noblet C. Trends in U.S. consumers'safe handling and consumption of food and their risk perceptions, 1988 through 2010. J Food Prot. 2011;74(9):1513-1523.

11. Kaferstein FK. Actions to reverse the upward curve of foodborne illness. Food Control 2003; 14(2): 101-109.

12. Lamuka PO. Public health measures: challenges of developing countries in management of food safety A2. In: Motarjemi Y, ed. Encyclopedia of food safety. Waltham, MA: Academic Press; 2014.

13. Makelele LK, Kazadi ZA; Oleko R W ; Foma R, Mpalang RA, Kabwang K. Microbiological quality of food sold by street vendors in Kisangani, Democratic Republic of Congo. African Journal of Food Science. 2015; 1 (9): 285-290

14. Alimi BA. Risk factors in street food practices in developing countries: A review. Food Science and Human Wellness (2016) 5: 141-148.

15. Green EJ, Knechtges PL. Food safety knowledge and practices of young adults. J Environ Health. 2015; 77(10):18-24.

16. Ferk CC, Calder BL, Camire ME. Assessing the food safety knowledge of university of Maine students. J Food Sci Educ. 2016;15(1):14-22.

17. Lazou T, Georgiadis M, Pentieva K, McKevitt A, Iossifidou E. Food safety knowledge and foodhandling practices of Greek university students: a questionnaire-based survey. Food Control. 2012;28(2): 400-411.
18. Hassan HF, Dimassi H. Food safety and handling knowledge and practices of Lebanese university students. Food Control. 2014; 40:127-133.

19. Majowicz SE, Diplock KJ, Leatherdale ST, Bredin CT, Rebellato S, Hammond D, et al. Food safety knowledge, attitudes, and self-reported practices among Ontario high school students. Can J Public Health. 2015;106(8):520-526.

20. Parry-Hanson Kunadu A, Ofosu DB, Aboagye E, Tano-Debrah K. Food safety knowledge, attitudes and self-reported practices of food handlers in institutional foodservice in Accra, Ghana. Food Control 2016; 69: 324-330.

21. Ministère de la santé et de lutte contre le SIDA; Côte d'Ivoire. Plan national de développement sanitaire 2013-1015, 92p.

22. Mohamed A, Waqas S, Oliyan S, Rayan S, Abdulrahman S, Nawaf R. Knowledge, attitude, and practice (KAP) of food hygiene among schools students' in Majmaah city, Saudi Arabia. J pak Med Assoc 2016, 66 4): 442-446.

23. Burton M, Cobb E, Donachie P, Curtis V, Judah G, Schmidt W-P. The effect of handwashing with water or soap on bacterial contamination of hands Int J Environ Res Public Health, 2011; 8: 97-104.

24. Curtis V, Cairncross S; Effect of handwashing with soap on diarrhea risk in the community: a systematic review. Lancet Infect Dis, 2003, 3, 275-281

25. Ali M, Verrill L, Zhang Y. Self-reported hand washing behaviors and food illness: a propensity score matching approach. J Food Protect, 2014; 77: 352-358.

26. Biran A, Rabie T, Schmidt W, Juvekar S, Hirve S, Curtis V. Comparing the performance of indicators of hand-washing practices in rural Indian households. Trop Med Int Health, 2008, 13 (2): $278-85$

27. Luby S, Halder A. Associations among handwashing indicators, wealth, and symptoms of childhood respiratory illness in urban Bangladesh. Trop Med Int Health, 2008, 13, 835-44.

28. Chauliac M, Bricas N, Ategbo E, Amoussa W, Zohoun I. [Food habits outside the home by school children in Cotonou (Benin)]. Sante. 1998 Mar-Apr;8(2):101-8

29. Chauliac M, Monnier T, Bendech MA. [Bamako school age children and their diet from street vendors].Sante. 1994 Nov-Dec;4(6):413-23. 
Tableau I : Caractéristiques socio-démographiques des élèves

\begin{tabular}{lcc}
\hline \multicolumn{1}{c}{ Age } & Effectifs & Pourcentage (\%) \\
\hline Médiane \pm EIQ & 133 & \\
9 à 14 ans & 232 & 29,7 \\
15 à 19 ans & 83 & 51,8 \\
20 à 25 ans & & 18,5 \\
\multicolumn{1}{c}{ Sexe } & 269 & 60,0 \\
Masculin & 179 & 40,0 \\
Féminin & & 2,9 \\
Conditions de vie & 416 & 92,9 \\
Vit chez un tuteur & & 12,7 \\
\hline Dispose d'argent de poche & & \\
\hline
\end{tabular}


Tableau II : Connaissances des élèves sur le lavage des mains, la gestion des déchets alimentaires, les maladies liées à la mauvaise hygiène alimentaire

Effectifs

Pourcentage

(\%)

\section{LAVAGE DES MAINS}

Méthode de lavage des mains
A l'eau simple
4
0,9
A l'eau et au savon
444
99,1

Technique de lavage des mains

Lavage des mains dans un récipient

Lavage des mains à partir d'une source d'eau qui

coule sur les mains

\section{GESTION DES DECHETS ALIMENTAIRES}

Lieu de stockage des déchets d'origine alimentaire

A même le sol

13

Dans une poubelle sans couvercle

Dans une poubelle à couvercle

$44 \quad 9,8$

391

\section{MALADIES LIEES A LA MAUVAISE HYGIENE} ALIMENTAIRE

Choléra

Fièvre typhoïde 
Tableau III : Pratique des élèves en hygiène des mains

\section{Effectifs}

425

Lavage systématique des mains avant chaque repas

Méthode de lavage des mains $(n=448)$

A l'eau simple 66

A l'eau et au savon

Technique de lavage des mains

Lavage des mains dans un récipient

Lavage des mains à partir d'une source d'eau qui coule sur les mains

Durée approximative du lavage des mains

Moins de 30 secondes

30 à 1 minute 
Tableau IV : Attitude des élèves des aliments vendus dans la rue

Effectifs

\section{Trouvaient malsaines}

La vente d'aliments à proximité d'ordures

La vente d'aliments à proximité d'eaux usées

La vente d'aliments à proximité de caniveaux ouverts

La présence de nuisibles aux points de vente d'aliments

Faisaient confiance aux aliments vendus
A l'école
Ni l'un ni l'autre

\section{Consommation d'aliments}

vendus à proximité d'ordures ménagères

vendus à proximité de caniveaux à ciel ouvert

vendus à proximité d'eaux usées

Pourcentage (\%)

393

87,7

419

424

94,6

421

Vendus non couverts

Déjà tombé malade après avoir consommé des aliments vendus dans la rue
30,8

53,6 